\title{
Trivium
}

Revue franco-allemande de sciences humaines et sociales - Deutsch-französische Zeitschrift für Geistesund Sozialwissenschaften

$8 \mid 2011$

Traductions et transferts des savoirs dans l'espace euro-méditerranéen à l'époque médiévale

\section{Von Antiochia bis Bagdad: Bibliotheken in Syrien und syrische Übersetzungen}

Javier Teixidor

Traducteur : Andreas Wittenburg

\section{OpenEdition}

Journals

Édition électronique

URL : http://journals.openedition.org/trivium/3928

DOI : 10.4000/trivium.3928

ISSN : 1963-1820

Éditeur

Les éditions de la Maison des sciences de l'Homme

\section{Référence électronique}

Javier Teixidor, « Von Antiochia bis Bagdad: Bibliotheken in Syrien und syrische Übersetzungen », Trivium [Online], 8 | 2011, online erschienen am 16 Mai 2011, abgerufen am 07 September 2020. URL : http://journals.openedition.org/trivium/3928 ; DOI : https://doi.org/10.4000/trivium.3928

Ce document a été généré automatiquement le 7 septembre 2020.

Les contenus des la revue Trivium sont mis à disposition selon les termes de la Licence Creative Commons Attribution - Pas d'Utilisation Commerciale - Pas de Modification 4.0 International. 


\title{
Von Antiochia bis Bagdad: Bibliotheken in Syrien und syrische Übersetzungen
}

\author{
Javier Teixidor
}

Traduction : Andreas Wittenburg

\section{NOTE DE L'ÉDITEUR}

Wir danken Javier Teixidor und den Editions de la BnF für die freundliche Genehmigung, diesen Artikel zu übersetzen.

1 Der arabische Buchhändler und Kopist an-Nadìm erzählt in seinem enzyklopädischen Werk, dem Fihrist, wie dem Kalifen al-Ma'mūn, dem jüngeren Sohn des Hārūn ar-Rašīd, eines Nachts ein Mann erschienen sei, der sich auf sein Bett gesetzt habe. Erschrocken habe der Kalif ihn gefragt, wer er sei, und mit großer Freude erfahren, dass es sich um keinen anderen als Aristoteles handelte. Nachdem er sich von seinem Erstaunen erholt habe, sei al-Ma'mūn in der Lage gewesen, einige Fragen zu stellen, auf die Aristoteles mit seiner üblichen Präzision geantwortet habe. Nach Darstellung an-Nadìms war dieser Traum der Anlass für das Interesse des Kalifen an den klassischen Autoren und an der Philosophie. Dieses Zusammentreffen habe ihn sogar dazu veranlasst, eine neue Politik gegenüber Konstantinopel einzuschlagen. In seinem fast einhundert Jahre nach dem Tod des Kalifen verfassten Buch listet an-Nadìm dem Leser dann die Bücher auf, die sein Vater, ein Buchhändler in Bagdad, hatte lesen und katalogisieren können, und dazu die Werke, die er selbst oder ihm Nahestehende gelesen haben. Auch wenn der Fihrist des an-Nadim vielleicht nicht so instruktiv ist wie die Schriften des Diogenes Laertios aus dem 3. Jahrhundert oder des Photios aus dem 9. Jahrhundert, bleibt das Werk doch eine wertvolle Quelle für die Kenntnis der Ideengeschichte im frühen Mittelalter. ${ }^{1}$ In ihm spiegelt sich die intellektuelle Welt des 9. und 10. Jahrhunderts wider. Natürlich stand nicht dieser Traum des Kalifen am Anfang des intellektuellen Lebens der Eliten in Bagdad; er war vielmehr die Bekräftigung einer Lehr- und 
Forschungstätigkeit, die schon seit mehreren Jahrzehnten bei einer größeren Zahl von jüdischen, christlichen und islamischen Gelehrten zu beobachten war. Bagdad bildete zu dieser Zeit das Zentrum der politischen und kulturellen Welt des Islam.

2 Seit ihren Anfängen war der Stadt eine glorreiche Zukunft bestimmt. Die Gründung Bagdads durch den abbasidischen Kalifen al-Manșūr im Jahre 762 gilt zu Recht als eines der wichtigsten Ereignisse in der Geschichte der islamischen Welt. Der arabische Geschichtsschreiber aț-Ṭabarī berichtet, wie al-Manșūr, an dem Ort seiner zukünftigen Hauptstadt angekommen, sein Abendgebet verrichtet habe. Am nächsten Tag habe er begriffen, dass der Ort dank des Euphrat und des Tigris und der zahlreichen Kanäle ein Verkehrsknotenpunkt werden könne, wie Babylon und Seleukeia-Ktesiphon es bereits waren. aț-Ṭabarī erzählt, dass al-Manșūr selbst den ersten Ziegel der Stadt gelegt und dabei ein Gebet gesprochen habe. Wer weiß, ob der Kalif und der Geschichtsschreiber sich dessen bewusst waren, dass dieser Gründungsakt die Wiederholung eines Rituals war, das in dieser Gegend der Welt seit über dreitausend Jahren bekannt war. al-Manș ūr wollte Bagdad zu einem von den syrischen Arabern unabhängigen politischen, wirtschaftlichen und kulturellen Zentrum machen. Er musste sich von der Politik der Umayyaden distanzieren, um sich auf die im Iran lebenden Araber stützen zu können; und ein Zeichen der neuen Politik war die Aufgabe der Verwaltungshauptstadt Wāsiț, die die Umayyaden für ihre Gouverneure des Irak errichtet hatten. Die Historiker sind sich darin einig, dass das iranische Element mit der Gründung von Bagdad größere Bedeutung erlangte. Schon der umayyadische Kalif Marwān II. hatte sich den Orientalen angenähert, als er seine Hauptstadt nach Ḥarrān im oberen Mesopotamien verlegt hatte. Die Abbasiden bekräftigten diese Politik, indem sie ihrer Staatsführung einen iranischen Anstrich gaben, dem die alten sassanidischen Verwaltungstraditionen nicht fehlten. Die geographische Lage Bagdads begünstigte diese Öffnung. Die Stadt befand sich nämlich am Ende einer traditionellen Verkehrsader über Hamad̄ān, Kermā nšāh und Hुāniqīn, die eine verhältnismäßig leichte Überquerung des Zagros erlaubte. Dieser Übergang war zu allen Zeiten benutzt worden, wie die große Inschrift von Behistun belegt, die Dareios in einen Felsen am Rande dieser Straße einmeißeln ließ, auf der Soldaten, Händler und Chronisten entlang zogen. ${ }^{2}$

Die Öffnung nach Osten war meiner Meinung nach nichts anderes als die konsequente Fortsetzung einer »Realpolitik«, die seit 363, als Kaiser Jovian die Gegend um Nisibis an die Sassaniden abgetreten hatte, von Römern und Persern verfolgt wurde. aț-Tabarī versichert, dass Schapur nach der Aufgabe von Nisibis durch die Römer zwölftausend Familien von Istakhr und Isfahan herangeführt und dort angesiedelt habe. ${ }^{3}$ Seine phantasievolle Erzählung steht im deutlichen Kontrast zum knappen Bericht des Ammianus Marcellinus, des Chronisten der römischen Niederlage. Man darf indes in Hinblick auf die weitere Entwicklung von Nisibis dieser Verpflanzung iranischer Bevölkerungsteile keine allzu große Bedeutung beimessen, denn das Syrische, das ein Dialekt des Aramäischen war, blieb die in der Stadt gesprochene und geschriebene Sprache. Alle Einzelheiten lassen darauf schließen, dass die offiziellen Strukturen der Kirche von Nisibis fast vollständig bestehen blieben und dass die Abfolge der Bischöfe unter den neuen Herren nicht unterbrochen wurde. Das Konzil von 410, das Isaak, der Bischof von Seleukeia-Ktesiphon, leitete, wurde mit einem feierlichen Gebet für den König Yazdegerd eröffnet. Die Schutzherrschaft der sassanidischen Staatsgewalt gab den Christen, wie es scheint, einen legitimen Status. ${ }^{4}$ Ein Orient war entstanden, der reicher an kulturellen Traditionen war als der Konstantinopels. Die wohlwollende 
Haltung der Kalifen gegenüber den christlichen Intellektuellen syrischer Sprache einige Jahrhunderte später stünde also im Rahmen einer festen politischen Tradition. Der Umstand, dass die Intellektuellen sich in ihrer Mehrzahl als Philosophen erweisen, die ich ohne zu zögern als praktizierende Aristoteliker bezeichnen würde, wirft die Frage auf, wie dieses intellektuelle Leben sich im Norden Mesopotamiens hatte entfalten können.

In diesem Zusammenhang ist unbedingt Antiochia zu erwähnen. Die Bedeutung dieser Stadt in den ersten Jahrhunderten unserer Zeitrechnung ist gut belegt. Sagt Flavius Josephus doch, dass Antiochia nach Rom und Alexandrien »wegen ihrer Größe und sonstigen Blüte unbestritten den dritten Rang unter den Städten des römischen Erdkreises einnimmt $\lll .{ }^{5}$ Prokopios nennt Antiochia die erste aller römischen Städte des Orients in Hinblick auf Reichtum, Größe und Bevölkerungszahl. ${ }^{6}$ Die Stadt erreichte im 3. Jahrhundert eine Bevölkerung von sechs- bis achthunderttausend Einwohnern. Das Christentum spielte hier schon früh eine Rolle, v. a. bei den Juden. Die Apostelgeschichte beschreibt den lebhaften Verkehr zwischen Jerusalem und Antiochia und die religiösen Differenzen zwischen den beiden Städten, und dann die Ankunft des Petrus in Antiochia, der sich für einige Zeit dort niederließ. Nach dem Niedergang Jerusalems überließ das Judenchristentum im gesamten Vorderen Orient seinen Platz einem hellenisierten Christentum, in dem die verschiedenen Strömungen des griechischen Denkens der Zeit Form annahmen. Antiochia war wie die meisten der großen Küstenstädte des Mittelmeerraums griechisch, aber sobald man die Stadt verließ, befand man sich mitten im semitischen Syrien. In den Vorstädten sprach man syrisch; in einer Homelie des Johannes Chrysostomus kann man lesen, dass die Bewohner dieser Vorstädte und der umliegenden Landbezirke, wenn sie in die Stadt kamen, zwar den Predigten beiwohnten, aber nur wenig davon verstanden. ${ }^{7}$

5 Auch wenn Antiochia seinen Ehrenplatz als die erste unter den großen Städten des Christentums infolge der Rivalität zwischen Konstantinopel und Alexandria verlor, wurde die Schule der Bibelauslegung, die der Priester Lukian am Ende des 3. Jahrhunderts dort gründete, ein Zentrum lebhaften intellektuellen Lebens. Im 4. und 5. Jahrhundert betrachteten sich vier herausragende Theologen als Schüler des Lukian: Diodor von Tarsus, Theodor von Mopsuestia, Theodoret von Cyrus und Nestorius, der Patriarch von Konstantinopel. Der Geschichtsschreiber Philostorgius nennt eine beeindruckende Liste von Persönlichkeiten, bei denen es sich ausnahmslos um Arianer der ersten Stunde handelt und die sich alle auch auf den antiochenischen Priester berufen. Bardy hat die Leitlinien der an dieser Schule praktizierten Auslegung des Bibeltextes folgendermaßen beschrieben:

»Unter den Kennzeichen der lukianischen Überarbeitung findet sich an erster Stelle ein großes Bemühen um Klarheit: Lukian fügt dem überlieferten Text eindeutige Begriffe hinzu, um den Sinn deutlicher werden $\mathrm{zu}$ lassen; er ersetzt Personalpronomen durch Eigennamen; er fügt neue Wörter ein, um eine dunkle Stelle zu erhellen; er füllt Lücken in der Erzählung aus. Manchmal scheint er sich dem hebräischen Text annähern zu wollen, aber das ist im Gegensatz zu Origenes nicht seine vordringliche Sorge. Es scheint ihm in erster Linie daran zu liegen, einen leicht $\mathrm{zu}$ lesenden und $\mathrm{zu}$ interpretierenden Text $\mathrm{zu}$ bieten, alle Zweideutigkeiten der Worte und auch all die Ungenauigkeiten auszuschließen, die nur durch Rückgriff auf die allegorische Exegese einen Sinn bekommen könnten. Solche Grundregeln stehen am Anfang einer wortwörtlichen Auslegung, die in der antiochenischen Schule auf Dauer in hohen Ehren stehen sollte, und vielleicht ist es 
deshalb, weil die Antiochener in der lukianischen Überarbeitung das Kennzeichen ihres eigenen Charakters sahen, dass sie sie vorrangig zu der ihren machten. ${ }^{8}$

Offensichtlich war es dieses »Kennzeichen ihres Charakters«, das zahlreichen Klerikern den Zugang zu den aristotelischen Texten und eine Öffnung hin zur Philosophie erleichterte. Mehrere Autoren syrischer Sprache widmeten sich vom 5. Jahrhundert an dem Studium dieser Philosophie, die in Athen wie in Alexandria von griechisch sprechenden neuplatonischen Philosophen aus dem 5. bis 7. Jahrhundert erklärt und ausführlich kommentiert wurde. Die syrischen Autoren, bei denen es sich fast ausschließlich um Kleriker handelte, gaben sich als Angehörige der Kirche von Antiochia zu erkennen, aber sie sprachen und schrieben nicht Griechisch, sondern Syrisch. So stark die Autorität Antiochias für diese Intellektuellen auch sein mochte, ihre begeisterte Aufnahme der aristotelischen Philosophie lässt sich eher aus dem Einfluss erklären, den Alexandria immer schon auf die Gegend von Antiochia ausgeübt hatte. Es sei daran erinnert, dass Zenobia von Palmyra erst Alexandria beherrschen und dann das Gebiet von Antiochia erobern wollte. Und von Eusebius von Caesarea wissen wir, dass in der politischen und religiösen Krise, die 268 in Antiochia durch die umstrittene Persönlichkeit des dortigen Bischofs Paul von Samosata ausgelöst wurde, Malchion, der Vorsteher »des Unterrichts in Rhetorik in den griechischen Schulen« der Stadt, darum gebeten wurde, sich über das Verhalten des Bischofs zu äußern. Doch seine Worte, so wohlgesetzt sie auch waren, hätten zur Verurteilung Pauls nicht ausgereicht, hätte nicht Anatolius von Alexandria eingegriffen, ein Mann von beeindruckender Gelehrsamkeit und großem Wissen, der aus Alexandria herbeigerufen worden war. Eusebius sagt über ihn: »In Arithmetik und Geometrie, in Astronomie und anderen Wissenschaften, Dialektik, Physik, Rhetorik hatte er es zu höchster Vollkommenheit gebracht und wurde daher, wie berichtet wird, von den Bürgern Alexandriens gebeten, dort die Schule aristotelischer Richtung zu gründen. $\ll^{9}$ Der Vorrang Alexandrias in der großen Kirche sollte später während der von den Arianern und Nestorianern hervorgerufenen theologischen Auseinandersetzungen noch deutlicher zu spüren sein.

7 Wir wissen, dass zwei sehr repräsentative Figuren der syrischen Schule, nämlich Sergius von Rēsh'ainā und Jakob von Edessa, einen Teil ihrer philosophischen Studien in Alexandria absolvierten. Die ersten syrischen Philosophen machten es sich zum Ziel, ihrer Sprache durch ihre Interpretation der aristotelischen Logik eine andere Dimension als die einer Umgangssprache zu geben. Das Syrische, das die Sprache einer alteingesessenen aramäischen Bevölkerung war, verzeichnete Vokale nicht, und das gab Anlass zur Sorge um eine korrekte Lesung des Bibeltextes. Die Kleriker waren gezwungen, zur Erleichterung der Rezitation den Text der Heiligen Schrift unter Einsetzung von Vokalen und anderen Zeichen zu fixieren. Die Grammatik half dabei zu lesen und zu schreiben, sie erklärte und interpretierte die Wörter; sie wurde die Grundlage sowohl der Erziehung wie dann der syrischen Kultur. Die syrischen Autoren drangen über die Auseinandersetzung mit dem Werk De interpretatione des Aristoteles auf das Gebiet der Grammatik vor. Der erste, der eine syrische Grammatik verfasste, war wohl Joseph Huzāyā im 6. Jahrhundert; sie ist die Wiedergabe eines entsprechenden Werks, das Dionys von Thrakien zugeschrieben wird. Die Sprache musste erklärt, die verwendeten Begriffe mussten gerechtfertigt werden, und vielleicht wurde die Sprache auf diese Weise zu einem Teil der Problematik, die sich mit den aristotelischen Kategorien befasst. Es ist durchaus möglich, dass Texte zur Logik und zur Grammatik von den syrischen Autoren in der Zeit nach dem 6. und 7. Jahrhundert 
gemeinsam studiert wurden, denn wie Hugonnard-Roche festgestellt hat, sind sie in manchen späten Handschriften »durch ihre Gegenüberstellung in parallelen Kolumnen $\aleph^{10}$ miteinander in Verbindung gebracht.

8 Der syrische Logiker Proba aus dem 6. Jahrhundert zeigt großes Interesse an De interpretatione: Für ihn stellt diese Abhandlung die Vollendung der Logik dar. Nach einer literarischen Technik, die später von Theodor Bar Kōnī verwendet werden sollte, war sein Kommentar in Form von Fragen und Antworten verfasst. Es ist gut möglich, dass jüdische Autoren wie Demetrius oder Aristobulos im 3. und 2. Jahrhundert v. Chr., um die Zeitenwende dann Philon von Alexandria diese Technik entwickelt hatten, um ihren Zeitgenossen die Bücher der Genesis und des Exodus zu erklären. ${ }^{11}$ Sergius von Rē sh'ainā, ein Zeitgenosse des Proba, war ein unermüdlicher Übersetzer des aristotelischen Corpus ins Syrische. Nach ihm gab es im 7. Jahrhundert vier große syrische Denker: Severus Sebokht, Athanasius von Balad, Georg Bischof der Araber und Jakob von Edessa. Mit ihrer Lehrtätigkeit und ihren Schriften machten diese Gelehrten aus den kirchlichen, bischöflichen oder monastischen Schulen von Edessa, Nisibis und Qinnasrīn, alle im oberen Mesopotamien gelegen, Zentren der biblischen Exegese, der Theologie und des Rechts, aber auch der profanen Wissenschaften wie Philosophie, Medizin oder Musik, die auf überzeugende Weise in das Lehrprogramm eingegliedert wurden. Die Bibliothek des Klosters von Qinnasrīn, wo Severus Sebokht wirkte, war reich an philosophischen und naturwissenschaftlichen Werken. Jakob von Edessa bezieht sich in seinem Kommentar über »die sechs Tage der Schöpfung" auf die zahlreichen Autoren, die er gelesen hat, aber leider nennt er niemals ihre Namen. Es ist erstaunlich, dass das intellektuelle Leben der syrischen Autoren fortbestehen konnte inmitten all der Umstürze, die sich im Laufe des 7. Jahrhunderts vollzogen, als sich zunächst Griechen und Perser und dann Griechen und Araber im westlichen Asien bekämpften. Der byzantinische Kaiser Herakleios, dessen Armee bis nach Ktesiphon vordrang, zwang den Persern im Jahre 630 den Frieden auf. Aber sein Erfolg hatte bekanntlich nur verheerende Auswirkungen auf Mesopotamien, wollte er doch die Juden und Monophysiten zur Annahme der Orthodoxie Konstantinopels zwingen. Mit Sicherheit betrachteten die Monophysiten die Ankunft der Araber als eine Befreiung. Die Schlacht am Yarmuk am 20. August 636 lieferte Syrien den Muslimen aus. Die Geschichtsschreiber berichten uns, dass die Bewohner von Damaskus den Arabern nach ihrem Sieg entgegengingen, um sie freudig willkommen zu heißen. Im Jahre 639 bemächtigten sich die Araber schließlich ganz Mesopotamiens.

Unsere Kenntnisse der syrischen Lehrstätten in der Periode zwischen der persischen und der arabischen Eroberung des Mittleren Ostens sind ziemlich unvollständig. Die Schule der Perser ist angeblich im 4. Jahrhundert von Ephrem in Edessa gegründet worden, als er das unter persische Herrschaft gefallene Nisibis verließ. Diese Nachricht wird von Barhadbeshabba, der im 6. Jahrhundert Bischof von Halwan war, in seiner Schrift Die Ursache für die Gründung der Schulen überliefert. ${ }^{12}$ Da Theodor von Mopsuestia als der offizielle Kommentator der Heiligen Schrift galt, wie uns Barhadbeshabba berichtet, musste man seine Schriften für die Schüler der neuen Schule aus dem Griechischen ins Syrische übersetzen. Der Übersetzer war Cyrus (Qiyōrē) von Edessa. Andere Historiker indes behaupten, es sei Ibas von Edessa gewesen. Für uns ist in diesem Zusammenhang wichtig, dass wir von Barhadbeshabba erfahren, dass Cyrus »betrübt" gewesen sei, weil die Kommentare des Theodor noch nicht ins Syrische übersetzt worden waren, aber dass die Übersetzung, sobald sie abgeschlossen war, »der Versammlung von Edessa vorgestellt wurde und Cyrus sich von der Versammlung 
bestätigt fühlte«. Mit den griechischen Texten des Theodor rückten auch die Werke des Aristoteles, des Porphyrius und Galens in den Blick. Der Isagoge des Porphyrius, zunächst ins Syrische und dann ins Arabische übersetzt, sollte während des ganzen Mittelalters unter den Intellektuellen des Vorderen Orients ein außerordentlicher Erfolg beschieden sein. Edessa wurde zu einem Zentrum der Studien, an dem man den theologischen Auseinandersetzungen in Antiochia aufmerksam folgte, doch die Spaltung in Monophysiten und Nestorianer sollte die Schule leider zu ihrem Niedergang führen. Die Notiz über Cyrus bei Barhadbeshabba gibt uns Auskunft über den Ausgangspunkt einer ganzen Geistesströmung, in der die syrischen Autoren mit Recht als Epigonen der griechischen Philosophie vor der Ankunft des Islam gelten.

Die Schule von Nisibis ist unter den syrischen Schulen vielleicht die uns am besten bekannte. Man wird sich erinnern, dass sich Nisibis seit 363 außerhalb des byzantinischen Einflussbereichs befand. Die Nachrichten über diese Schule sind von besonderer Bedeutung, weil sie das intellektuelle Leben im sassanidischen Reich direkt betreffen. Der Bischof von Nisibis im 5. Jahrhundert, Barsauma, ist eine herausragende Figur der persischen Kirche. Seine verschiedenen Aktivitäten kennen wir aus dem Brief, den der im Dienste Konstantinopels stehende Simeon von Beth Arsham im 6. Jahrhundert über die nestorianischen Kirchen im persischen Gebiet verfasste. ${ }^{13}$ Unter den politischen und literarischen Unternehmungen des Barsauma kann seine Gründung der sich zum Nestorianismus bekennenden Schule von Nisibis vielleicht als die bemerkenswerteste Tat gelten, wenn man an den Einfluss denkt, den sie im Mittelalter im Orient und selbst im Westen gehabt hat. Mit den folgenden Worten überredete Barsauma den wegen seines nestorianischen Glaubens aus Edessa vertriebenen Narsai dazu, in Nisibis eine Schule wie die von Edessa zu gründen: »In ganz Persien werdet Ihr keine Stadt finden können, die für Euch besser geeignet ist als diese; es ist eine wichtige Stadt; und, da sie zwischen zwei Großreichen gelegen ist, kommt man aus allen Gegenden dorthin; wenn man erfahren wird, dass es hier eine Schule gibt und, vor allem, dass Ihr es seid, der sie leitet, wird man sich in Scharen um Euch drängen. « ${ }^{14}$ Barsauma half Narsai, diese Schule zu gründen, die in der Tat ebenso bedeutend wurde wie die von Alexandria und am Ende eine entscheidende Rolle für die Verbreitung der hellenistischen Kultur im Westen spielte. Das Ansehen der Schule von Nisibis verbreitete sich sogar bis in das Zentrum des Westreichs. Magnus Aurelius Cassiodorus, der zuvor Präfekt unter Theoderich gewesen war, zog sich vom politischen Leben zurück und gründete in seinem Heimatland neben Klöstern auch eine dazugehörige Akademie: Es handelt sich hier um Vivarium, einen angesehenen Ort des Studiums und der schriftstellerischen Tätigkeit. Cassiodorus hatte den Papst Agapetus gebeten, eine Schule gründen zu dürfen wie die von Nisibis, die er während eines kurzen Aufenthalts im Norden Mesopotamiens besucht hatte. ${ }^{15}$ Die Schule von Nisibis gab sich offen und kämpferisch konfessionell, d. h. in ihrem Fall nestorianisch. Dieser kämpferische Aspekt wurde von den Juden der Stadt unterstützt, die sich zumindest bei einer Gelegenheit zu Beginn des 8. Jahrhunderts mit den Nestorianern verbündeten, um die Kirche der Monophysiten zu zerstören. Nach Aussage einer syrischen Chronik aus dem 9. Jahrhundert musste Mar Simeon, der Bischof von Ḥarrān, die »orthodoxe« (d.h. monophysitische) Kirche von Nisibis dreimal wiederaufbauen, weil die am Morgen verrichtete Arbeit am Abend von den Nestorianern und den Juden wieder zerstört worden war. ${ }^{16}$ Chosrau I. Anuschirwan, der 531 an die Macht kam, dem Einfluss des Hellenismus, und 
dank der Protektion dieses sassanidischen Königs, die die Gegend genoss, schrieb Paul der Perser, der angeblich in Nisibis studiert hatte, eine Zusammenfassung der Logik des Aristoteles auf Syrisch, die er Chosrau widmete. ${ }^{17}$ Der arabische Philosoph Aḥmad b. Miskawayh (10. Jahrhundert) zitiert in seiner Abhandlung über das Glück einen langen Text des Paul, in dem Letzterer mehr Aufschluss über seine Kenntnisse des Aristoteles gibt als in dem Chosrau gewidmeten Text. Die Einteilung der Wissenschaften, die Paul dort vorschlägt, deckt sich mit den Lehren der Schule von Alexandria und geht vielleicht auf den Einfluss des David zurück, eines auf Griechisch schreibenden armenischen Philosophen des 6. Jahrhunderts. Nach der armenischen Überlieferung soll David die Philosophie in Athen und Konstantinopel studiert haben. Neuere Untersuchungen erlauben den Schluss, dass er in Alexandria Schüler des jüngeren Olympiodorus war. David unterscheidet zwischen den Gegenständen der theoretischen Philosophie - Engel, Gott, Seele - und denjenigen, die steter Veränderung unterliegen. Ohne Zweifel geht es auf David zurück, wenn Paul der Perser dieselbe Einteilung vornimmt: einerseits die erkennbaren Wesen - Seele, Dämonen, Engel -, andererseits die Dinge der Natur, die entstehen und alsbald wieder vergehen. Paul war dem Rat Davids gefolgt, der hervorgehoben hatte, dass der Leser des Aristoteles sein ganzes Werk kennen solle, sodass »er, nachdem er gezeigt hat, dass Aristoteles immer mit sich selbst übereinstimme, das Werk des Aristoteles aus dem Werk selbst erklären könne«. ${ }^{18}$

Am Ende des 8. Jahrhunderts ist Theodor Bar Kōnī sich zwar der Rolle bewusst, die die Logik als Fundament der Grammatik spielt, aber er führt nicht im Einzelnen aus, was die beiden Disziplinen miteinander verbindet. Die Polemiken, die sich arabische Logiker und Grammatiker geliefert hatten, scheinen bei den syrischen Autoren nicht stattgefunden zu haben. ${ }^{19}$ Die Bedeutung, die die syrischen Autoren dem Verbum als Begriff zumessen, lässt sich damit erklären, dass sie dem aristotelischen Text von $D e$ interpretatione verhaftet sind. Man kann sich aber nicht sicher sein, ob sie sich den Bemerkungen angeschlossen hätten, die der Philosoph Mattā b. Y ūnus in der berühmten um 940 in Bagdad ausgetragenen Kontroverse dem Grammatiker Abū Sa ${ }^{c} \bar{d}$ as-Sīrāfī entgegengehalten hat: »Von eurer Sprache brauche ich nichts anderes als das Substantiv, das Verb und die Partikel, um die Gedanken auszudrücken, die Griechenland für mich geordnet hat. « ${ }^{20}$

Im Rahmen einer Lektüre der Abhandlung zur Logik Pauls des Persers und der Schriften anderer zeitgenössischer oder späterer Philosophen kann man nach und nach den Beitrag der syrischen Autoren zur Ideengeschichte ermessen. Diese christlichen Philosophen, die fast alle Kleriker sind und doch glücklicherweise alle theologischen Kontroversen beiseitelassen, haben in ihrer eigenen Sprache Kommentare $\mathrm{zu}$ Aristoteles verfasst und dabei dieselbe Begeisterung und dieselbe Leidenschaft für Wissen an den Tag gelegt wie ihre Zeitgenossen an den Schulen von Alexandria und im Norden Syriens. Man kann von diesen syrischen Autoren sagen, dass es nicht ihr Ziel war, ein eigenes philosophisches System zu errichten, sondern vielmehr die Wahrheit zu verkünden, wie sie die griechischen Philosophen erwiesen hatten. Mehr und mehr versteht man nun die Interpretation, die diese Gruppe von Autoren syrischer Sprache von den aristotelischen Texten gegeben haben, stammten sie doch alle aus einer Gegend, die in der Spätantike ein wahrer Schnittpunkt der Kulturen war. Diese Intellektuellen entsprachen der Beschreibung des Barhadbeshabba von Halwan aus dem 7. Jahrhundert, wenn er in einem beeindruckenden Text in der Einleitung zu 
seinem bereits zitierten Werk Die Ursache für die Gründung der Schulen die intellektuelle Kraft des Menschen beschreibt. Der Intellekt hat

»die Kraft, zum Himmel und zur Höhe der Kuppeln aufzusteigen; und dort (ist er) wie in den Palästen des Königreichs und den himmlischen Hallen, er durchstreift (dann) die Basare und die ausgedehnten Plätze, die sich unter den oberen Himmelsgefilden befinden. Bisweilen, um sich zu erholen, steigt er in den weiten Raum zwischen dem Firmament und dem Himmel hinab und bleibt dort allein und von der Seele getrennt, als (ob er sich) in einer königlichen Residenz (befände). Wenn er will, dringt er von dort in den Bereich der Erde vor, der sich unter dem Firmament befindet; er fliegt in dieser Region des Feuers umher und verbrennt sich nicht, er schreitet auf den Sternen umher wie auf Steinen in einem Fluss und versinkt nicht. [...] Und da er von Zeit zu Zeit seine Gedanken auf den Lauf der Sonne, die Phasen des Monds und die Ordnung der Sterne richtet ... Sein Herr gibt ihm dann und wann Macht über diese Gestirne, damit sie sich nach ihrer Ordnung bewegen, wie wir es bei Josua, dem Sohn Nuns sehen, der eines der Gestirne (die Sonne) über Gibeon angehalten hat und das andere (den Mond) im Tal Ajalon.« Der Intellekt ist vom Schöpfer »in den weiten Raum gestellt, der sich zwischen Himmel und Erde auftut. Er hat sozusagen alle sichtbaren Körper auf einer Tafel aufgezeichnet, damit die Einsicht der Verstandeskraft sie dort lesen kann. «11

Barhadbeshabba scheint an die Präexistenz der Verstandeskräfte zu glauben. Sein Text weist auf die Zeit zurück, in der die Philosophie des Aristoteles waus der Sicht der Lehren Platons" erklärt wurde, »die ihrerseits im Lichte der neuplatonischen Philosophie neu interpretiert worden war", wie Philippe Hoffmann überzeugend gezeigt hat. ${ }^{22}$

Das Bagdad des al-Manșūr war Erbe dieser kulturellen Welt. Euphrat und Tigris verbanden es mit Mosul und dem Norden Mesopotamiens, wo die syrischen Klöster zu Zentren der griechischen Kultur geworden waren. Neben den Bibelkommentaren übersetzte man die philosophischen und naturwissenschaftlichen Werke der Antike. Ein wichtiger Text des Ibn Haldūn (1332-1406) in seinem Werk al-Muqadimma gibt uns folgenden Bericht:

»Am Anfang waren die Araber einfache Menschen ohne Interesse an den Künsten. Aber einhergehend mit der Entwicklung des Staates nahmen sie nach und nach eine sesshafte Lebensweise an, wie sie bis dahin niemand gekannt hatte. Sie machten sich mit zahllosen Künsten und zahllosen Wissenschaften vertraut. Dann wollten sie die philosophischen Wissenschaften studieren. Sie hatten die Bischöfe und Priester ihrer christlichen Untertanen davon sprechen hören, und im Übrigen fühlt sich das Denken des Menschen ganz allgemein dazu hingezogen zu lernen. Aus diesem Grunde ließ al-Manșūr den Kaiser von Byzanz darum bitten, ihm (arabische) Übersetzungen der Bücher über Mathematik zuzusenden. Der Kaiser ließ ihm die Abhandlungen des Euklid und einige Werke zur Naturgeschichte bringen. Die Muslime lasen und studierten all diese Werke, und das weckte bei ihnen die Lust, noch mehr zu lernen. « $^{23}$

Die Christen erfreuten sich offensichtlich eines gewissen Ansehens und hatten sogar Einfluss auf die muslimischen Eliten. Der zitierte Text erlaubt die Annahme, dass es zahlreiche Kontakte zwischen Christen und Muslimen gab. Im Jahre 644 hat nach der Chronik Michaels des Syrers (II, 432) 'Amru, der Sohn des qurayšitischen Gouverneurs von Medina Sa'd, den monophysitischen Patriarchen Johannes aufgefordert: »Übersetze mir euer Evangelium in die arabische Sprache. Aber du sollst weder von der Göttlichkeit Christi, noch von der Taufe oder dem Kreuz sprechen." Der Text wurde übersetzt, immer nach Aussage der Chronik, und zwar unter Mithilfe »von Angehörigen der arabisch-christlichen Stämme Mesopotamiens, die das Arabische und das Syrische beherrschten «. ${ }^{24}$ Eineinhalb Jahrhunderte später fand der nestorianische Patriarch 
Timotheus Gelegenheit, erst in Ktesiphon und dann in Bagdad Beziehungen mit dem Kalifen al-Mahdī zu unterhalten. In einem seiner Briefe schrieb Timotheus Folgendes:

»Eine königliche Anordnung (des berühmten Hārūn ar-Rašīd) hat von uns das Buch über die Topik des Philosophen Aristoteles verlangt, das wir aus dem Syrischen ins Arabische übersetzen sollten, und das wurde mit der Hilfe Gottes und unter Beteiligung des Meisters Abū N ūḥ [ein nestorianischer Autor, Sekretär des muslimischen Gouverneurs von Mosul] vollendet. Was unsere Arbeiten betrifft, so haben sie die volle Zustimmung des Kalifen gefunden, besonders als er sie Stück für Stück mit den anderen verglichen und bewertet hat. Eure Heiligkeit suche also mit Umsicht in Erfahrung zu bringen, ob es über dieses Buch über die Topik oder über das, das die Sophisten widerlegt, oder über die Rhetorik oder die Poetik einige Kommentare oder Scholien von irgendwelchen Autoren auf Syrisch gibt oder nicht. Wenn es welche gibt, findet heraus, von wem sie sind, wem sie gehören und wo sie $\mathrm{zu}$ finden sind. $\aleph^{25}$

Nach al-Manșūr setzte der Kalif al-Ma'mūn die gleiche Kulturpolitik fort und entsandte ebenfalls Gesandtschaften an den Hof von Byzanz. Ibn Haldūn fügt hinzu, dass der Kalif eine Arbeitsgruppe von Übersetzern geschaffen habe, die aus Gelehrten bestand, die die aus Byzanz eintreffenden griechischen wissenschaftlichen Werke ins Arabische übersetzen sollten. Der arabische Historiker bemerkt:

"Sie zeichneten sich so sehr in den verschiedenen Disziplinen aus, dass niemand es hätte besser machen können. Sie gingen sogar so weit, den ersten Meister (Aristoteles) in mehreren Punkten zu kritisieren. Aber seines Ruhms wegen hielten sie ihn für die höchste Autorität: sie verließen sich auf ihn, wenn sie wissen wollten, ob sie eine Meinung übernehmen oder zurückweisen sollten. Sie schrieben darüber ganze Abhandlungen und übertrafen ihre Vorgänger in den geistigen Wissenschaften. $\ll^{26}$

$\mathrm{Zu}$ ebendieser Zeit wurde aus der Bibliothek, von der an-Nadīm spricht und die schon beim Herrschaftsantritt des al-Ma'mūn bestanden hatte, ein Zentrum der Studien. Im 9. Jahrhundert war Ḥunayn b. Isḥāq, ein nestorianischer Christ syrischer Sprache, die beherrschende Figur dieses Zentrums. Der 809 in Hīra geborene Sohn eines Apothekers interessierte sich von Jugend an für Medikamente und die Medizin, darunter besonders für Ophtalmologie. Er erwarb seine Kenntnisse bei Yuhannā b. Māsawayh, der in Europa unter dem Namen Johannes Mesue bekannt wurde, einem Arzt aus der Schule von Gundishapur, der unter mehreren Kalifen am Hof der Abbasiden diente. Seine Werke wurden 1471 in Venedig und 1478 in Lyon übersetzt. Die Überlieferung will, dass Hunayn aus der Schule vertrieben wurde, weil er zu viele unangenehme Fragen stellte. Für zwei Jahre verschwand er aus der Hauptstadt. Die Geschichtsschreiber berichten uns, dass er in Alexandria Griechisch gelernt und in Basra im Irak seine Kenntnisse des Arabischen vervollständigt habe. Die arabischen Historiker kennen ihn als Arzt im Dienst des Kalifen al-Mutawakkil, ansonsten ist er aber vor allem als Linguist und Übersetzer bekannt. In einem Brief an 'Alī b. Yahyā behauptet er, er habe im Alter von siebzehn Jahren ein Werk des Galen über die Gewalt der Naturkräfte aus dem Griechischen ins Syrische übersetzt. Fast dreißig Jahre später spricht er davon:

»Als ich über vierzig Jahre alt war, bat mich mein Schüler Hubayš, die griechische Fassung zu korrigieren, nachdem ich eine gewisse Anzahl anderer Handschriften desselben Textes beschafft hatte. Ich habe all diese Handschriften verglichen, um einen korrekten Text zu erstellen, und habe dann diesen Text mit meiner (ersten) syrischen Übersetzung verglichen, um diese zu berichtigen. So gehe ich bei all meinen Übersetzungen vor. $\aleph^{27}$ 
Auf mehreren Reisen im Irak, in Syrien, nach Alexandrien und nach Byzanz suchte $\dot{H}$ unayn nach Texten, die er übersetzen konnte. Wir verdanken ihm eine Liste der verschiedenen zu seiner Zeit zugänglichen Übersetzungen des Galen. Sein kritisches Urteil findet in der modernen Textkritik volle Zustimmung. ${ }^{28}$ Eine bemerkenswerte Charakteristik dieser dauerhaft vom kritischen Geist Hunayns erfüllten Schule von Übersetzern war ihre Überzeugung, dass die Übersetzung eines Texts niemals als endgültig gelten kann. An-Nadìm erinnert mehrmals daran. Oft überprüfte man die übersetzten Texte, wobei die Revision manchmal einem anderen Gelehrten übertragen wurde. Sicher hielt man diese ständige Überprüfung der Übersetzungen für notwendig, weil das Arabische nicht immer die Muttersprache des Übersetzers war, aber sie stellte doch ebenso eine wohlüberlegte Vorgehensweise dieser Schule von Übersetzern dar. Die erhaltenen Zeugnisse unterrichten uns darüber, dass man häufig eine Zusammenfassung des $\mathrm{zu}$ übersetzenden Textes anfertigte, wofür eine vorherige Beherrschung des in Frage stehenden Themas notwendig war. Die Kompetenz und Strenge der Arbeit der Übersetzer von Bagdad können noch heute als Vorbild dienen. Nestorianische Kleriker, Rabbiner und muslimische Theologen, Ärzte, Mathematiker, Astronomen und Logiker scheinen allesamt an einem kulturellen Unterfangen mitgewirkt zu haben, dessen Bedeutung für die allgemeine Kulturgeschichte immer bestehen bleibt.

\section{BIBLIOGRAPHIE}

\section{Quellen}

aț-Ṭabarī, Annales, Ed. Barth /Nöldeke, in: Dodgeon / Lieu (1991).

Anecdota syriaca, Ed. und übers. J. P. N. Land, Paris 1875.

Barhadbeshabba 'Arbaya, La cause de la fondation des écoles, Ed. und übers. Addai Scher (Patrologia Orientalis 4), Paris 1908, S. 319-404.

Barhadbeshabba 'Arbaya, Histoire nestorienne, Ed. Addai Scher, 2. Teil (Patrologia orientalis 7), Paris 1911, S. 92-203.

Barhadbeshabba 'Arbaya, Histoire ecclésiastique, Ed. F. Nau (Patrologia orientalis 9), Paris und Freiburg i. Br. 1913, S. 493-631.

Chronicon anonymum ad annum Domini 819 pertinens, Ed. Aph. Barçauma, übers. Jean Baptiste Chabot (CSCO ser.3, t. 14), Paris 1920.

Eusebius von Caesarea, Kirchengeschichte, Ed. Heinrich Kraft, übers. Philipp Haeuser, 2. Aufl., München 1981.

The Fihrist of al-Nadìm, Ed. und übers. B. Dodge, 2 Bde., New York 1970.

Flavius Josephus, Der jüdische Krieg, übers. Philipp Kohout, Linz 1901; http://de.wikisource.org/ wiki/Juedischer_Krieg (13.2.2011) 
Patrologia latina, Ed. Jacques Paul Migne, Bd. 70, 1105.

Procopios, De bello Persico

Ibn Khaldūn, Al-Muqadimma, übers. V. Monteil, Paris 1997; Beirut 1967-1968.

Simeon von Beth Arsham, in: Assemani, J. S. (1719); Ortiz de Urbina, I. (1965).

\section{Sekundärliteratur}

Arabica IX, Volume spécial publié à l'occasion du mille deux-centième anniversaire de Baǵdad, Leiden 1962.

Arnaldez, R. (1962): „Sciences et philosophie dans la civilisation de Bagdâd sous les premiers >Abbâsides«", Arabica, IX, S. 357-373.

Assemani, J. S. (1719-28): Bibliotheca orientalis Clementino-Vaticana, 4. Bde., Rom.

Bardy, G. (1929): Paul de Samosate, Louvain.

Bergsträsser, G. (1925): Über die syrischen und arabischen Galen-Übersetzungen, Leipzig.

Charon, C. (1908): Echos d'Orient 11, S. 82-89.

de Vaumas, E. (1962): »Introduction géographique à l'étude de Badgad«, Arabica, IX, S. 229-246.

Dodgeon, M. H. / Lieu, S. N. C. (1991): The Roman Eastern Frontier and the Persian Wars, AD 226-363, London.

Elamrani-Jamal, E. (1983): Logique aristotélicienne et grammaire arabe (Etudes et documents), Paris.

Georr, Kh. (1948): Les Catégories d'Aristote dans leurs versions syro-arabes, Beirut.

Goulet, R. (Hg.) (1989): Dictionnaire des philosophes antiques, Bd. 1, Paris.

Helm, R. (1954): Reallexikon für Antike und Christentum, Bd. 2, Stuttgart 1954, col. 919 s.v. »Cassiodorus«.

Hoffmann, Ph. (1996): „L'arrière-plan néoplatonicien de l'Ecole d'Athènes de Raphaël«, in: Hoffmann, Ph. / Rinuy, P.-L. (Hg.) (1996): Antiquités imaginaires. La référence antique dans l'art moderne, de la Renaissance à nos jours, Paris, S. 143-158.

Hugonnard-Roche, H. (1991): „L'intermédiaire syriaque dans la transmission de la philosophie grecque à l'arabe : le cas de l'Organon d'Aristote«, Arabic Sciences and Philosophy, 1, S. 187-209.

Hugonnard-Roche, H. (1994): „Les traductions syriaques de l'Isagoge de Porphyre et la constitution du corpus syriaque de logique«, Revue d'histoire des textes, 24, S. 293-312.

Nau, F. (1929): „L'araméen chrétien (syriaque). Les traductions faites du grec en syriaque au VIIe siècle«, Revue de l'histoire des religions, 99, 2/3, S. 232-287.

Ortiz de Urbina, I. (1965): Patrologia syriaca, 2. Aufl., Rom.

Palmer, A. (1993): The Seventh Century in the West-Syrian Chronicle, Liverpool.

Renan, E. (1852): »Lettre à M. Reinaud, sur quelques manuscrits syriaques du Musée britannique«, Journal asiatique $4^{\mathrm{e}}$ série, 19, S. 311-319.

Sourdel, D. (1999): L'État impérial des califes abbassides, Paris.

Strohmaier, G. (1971) : Encyclopédie de l'Islam Bd. 3, 1971, S. 598-601. 
Teixidor, J. (1995): „Conséquences politiques et culturelles de la victoire sassanide à Nisibe«, in: Frezouls, Ed. / Jaquemin, A. (Hg.) (1995): Les relations internationales (Actes du Colloque de Strasbourg, 15-17 juin 1993), Strasbourg.

Teixidor, J. (1996-1997): »Aristote en syriaque : les philosophes de la haute Mésopotamie au VIe siècle«, Annuaire du Collège de France. Résumé des cours et travaux 1996-1997, Paris, S. 723-741.

Teixidor, J. (1998) : «L'introduction au De intrepretatione chez Proba et Paul le Perse », in : Lavenant, R. (Hrsg.) (1998) : Or.Chr.Ann.256, Symposium Syriacum VII, Rom, S. 301.

Wiatkowski, W. (1987): The Syriac Chronicle of Pseudo-Dionysus of Tel-Mahre, Uppsala.

\section{NOTES}

1. Fihrist 7, 1, Bd. 2. S. $583-584$.

2. Vgl. dazu die Bemerkungen bei de Vaumas (1962) in dem aus Anlass des 1200. Jubiläums der Stadtgründung veröffentlichten Sonderband der Zeitschrift Arabica über Bagdad, S. 233-236; in demselben Band, S. 357, bemerkt R. Arnaldez: »Die geographischen Schriftsteller, die den Ruhm Bagdads verkündet hatten, und darunter besonders al-Muqaddasi und al-Ya'qūbī, waren unerschöpflich in ihren Lobpreisungen Bagdads. Dass der Ort gesegnet sei, war dem Propheten offenbart worden. Die Luft dort war rein und gesund, der Boden fruchtbar, die Temperaturen der verschiedenen Jahreszeiten waren gemäßigt. Die Einwohner verfügten über einen wachen Geist und waren begabt in Hinblick auf die Wissenschaften und die verschiedensten Spekulationen. Die Verbindungswege zu Wasser und zu Lande liefen an diesem Zentrum zusammen, das wahrlich der Nabel der Welt war, und brachten alle materiellen und geistigen Reichtümer der Welt dorthin.« Vgl. dazu auch Sourdel (1999), S. 33-43.

3. aț-Tabarī, Annales, S. 294.

4. Teixidor (1995), S. 505.

5. Flavius Josephus, Der jüdische Krieg 3, 2, 4.

6. Procopios, De bello persico 1, 17.

7. Bardy (1929), S. 203. Bardy zitiert den Beleg bei Sokrates, der im Zusammenhang mit Severianus von Gabala sagt, es habe Bischöfe gegeben, die Griechisch mit einem starken syrischen Akzent gesprochen hätten; vgl. dazu auch Charon (1908), S. 82-89, über das Fortbestehen der syrischen Sprache und des syrischen Geistes in Antiochia und in der Umgebung der Stadt.

8. Bardy (1929), S. 381-382.

9. Eusebius, Kirchengeschichte 32, 6-20 (übers. v. Philipp Häuser). Eusebius beschreibt die politische Persönlichkeit des Anatol sehr zutreffend; zu dessen wissenschaftlicher Tätigkeit vgl. zuletzt Goulet (1989), S. 179-183.

10. Hugonnard-Roche (1994), S. 309.

11. Teixidor (1998), S. 301.

12. Barhadbeshabba 'Arbaya, La cause de la fondation des écoles, Ed. und Übers. Addai Scher (Patrologia Orientalis 4), Paris 1908, S. 319-404. Angemerkt sei, dass das Wort für »Schule«, eskūlā , aus dem Griechischen kommt.

13. Simeon vertrat die Politik der Zentralkirche von Konstantinopel; er entfaltete seine Tätigkeit gegen die Nestorianer auf persischem Gebiet. Assemani (1719), S. 346-358; Ortiz de Urbina (1965), S. 162.

14. Barhadbeshabba 'Arbaya, La cause de la fondation des écoles, S. 384-385. Eine zusätzliche Bemerkung in Barhadbeshabba 'Arbaya, Histoire ecclésiastique, S. 605 [117]-606 [118] und in Barhadbeshabba 'Arbaya, Histoire nestorienne, S. 114 [22]. Die militärische Bedeutung Nisibis für 
die Perser ist in der Ansprache des Belisar an seine Soldaten treffend beschrieben: Procopios, De bello Persico 2, 18, 1-15.

15. Patrologia latina, Bd. 70, 1105. Vgl. dazu Helm (1954), Sp. 919.

16. Chronicon anonymum ad annum Domini 819 pertinens; Palmer (1993), S.79, und Witakowski (1987), S. 4, 57.

17. Anecdota syriaca, Bd. 4, S. 1-32, mit einer lateinischen Übersetzung. Vgl. dazu Renan (1852), S. 311-319. Ich verstehe nicht, warum diese Abhandlung zuerst auf Persisch verfasst und dann von Severus Sebokht ins Syrische übersetzt worden sein soll, wie Georr (1948), S. 25, behauptet; man sprach Syrisch in Mesopotamien; vgl. dazu Anecdota syriaca, S. 101.

18. Vgl. dazu Teixidor (1996-1997), insbesondere S. 733-734.

19. Das Buch von Elamrani-Jamal (1983) gibt eine gute Vorstellung von den damaligen akademischen Auseinandersetzungen.

20. Text bei Elamrani-Jamal (1983), S. 154.

21. Barhadbeshabba 'Arbaya, La cause de la fondation des écoles, S. 334; S. 344-347.

22. Hoffmann (1996).

23. Ibn Khaldūn, Al-Muqadimma, S. 803-804.

24. Zitiert nach Nau (1929), S. 283-284.

25. Nau (1929), S. 284; vgl. dazu Hugonnard-Roche (1991), insbesondere S. 197.

26. Ibn Khaldūn, Al-Muqadimma, S. 804.

27. Übersetzung von M. Salama-Carr des bei Bergsträsser (1925) edierten arabischen Texts (mit deutscher Übersetzung).

28. Zu Ḥunayn vgl. Strohmaier (1971), S. 598-601.

\section{INDEX}

Mots-clés : Aristote, Antioche, Alexandrie, philosophes syriaques, école de Nisibe

Schlüsselwörter : Aristoteles, Antiochia, Alexandria, syrische Philosophen, Schule von Nisibis

\section{AUTEURS}

\section{JAVIER TEIXIDOR}

Javier Teixidor war bis 2001 Honorarprofessor am Collège de France. Nähere Informationen

finden Sie hier. 